\title{
DISTÚRBIOS DA COMUNICAÇÃOO ORAL EM CRIANÇAS
}

\author{
ORAL COMMUNICATION DISTURBANCES IN CHILDREN
}

Márcia Madeira Peres de Vitto ${ }^{1}$, Maria Cristina Lancia Cury Feres²

\begin{abstract}
${ }^{1}$ Médica otorrinolaringologista.Faculdade de Medicina de Catanduva. ${ }^{2}$ Docente. Divisão de Otorrinolaringologia. Departamento de Oftalmologia, Otorrinolaringologia e Cirurgia de Cabeça e Pescoço.Faculdade de Medicina de Ribeirão Preto-USP.

Correspondência: Profa. Dra. Maria Cristina Lancia Cury Féres. Departamento de Oftalmologia, Otorrinolaringologia e Cirurgia de Cabeça e Pescoço.Divisão de Otorrinolaringologia do Hospital das Clínicas de Ribeirão Preto. Avenida Bandeirantes, 3900. Campus Universitário - Monte Alegre, 14.048-900 - Ribeirão Preto - SP Fone: (16) 3602-2863 - Fax: (16) 3602-2860 ccury@fmrp.usp.br; mcferes@hcrp.fmrp.usp.br
\end{abstract}

Vitto MMP, Féres MCLC. Distúrbios da comunicação oral em crianças. Medicina (Ribeirão Preto) 2005; 38 (3/4): 229-234.

RESUMO: Os distúrbios da comunicação oral em crianças são muito comuns, podendo ser causados por diversos tipos de alterações, incluindo distúrbios auditivos, neurológicos e psiquiátricos. É de grande importância o diagnóstico precoce da origem do problema, para intervenção terapêutica apropriada, a fim de minimizar os déficits decorrentes desses distúrbios. Os autores apresentam uma revisão sobre o assunto.

Descritores: Transtornos da Comunicação. Transtornos da Linguagem. Distúrbios da Fala.

\section{1- INTRODUÇÃO}

Os distúrbios no desenvolvimento da linguagem e da fala são muito comuns, afetando 5 a $10 \%$ de todas as crianças. Estima-se que $1 \%$ das crianças vêm para a escola com uma deficiência de linguagem marcante. $\mathrm{O}$ atraso na linguagem pode ter um significante impacto na vida social e escolar, e sua identificação precoce, com intervenção apropriada, podem atenuar os déficits emocional, social e cognitivo.

\section{2- COMUNICAÇÃO ORAL}

Comunicação, do latim communicatio, pode ser definida como um meio pelo qual o indivíduo relata experiências, idéias, conhecimentos e sentimentos a um outro. A mensagem a ser compartilhada é codificada na fonte e decodificada no destino, com o uso de sistemas de signos ou símbolos sonoros, iconográficos ou gestuais, entre outros ${ }^{1}$.
A linguagem é um desses sistemas; os símbolos da linguagem, quando arranjados em particular ordem, podem ser usados para exprimir pensamentos, idéias, desejos e emoções. A linguagem pode ser estudada através da semântica (estuda quais palavras que são escolhidas, o repertório), a sintaxe (estuda a ordem das palavras), a fonologia (estuda o som das palavras) e a morfologia (estuda a forma das palavras). Esse sistema de símbolos usados para a comunicação interpessoal é um aprendizado cultural, estando fortemente ligado ao ambiente de convívio do indivíduo.

A forma mais evidente de expressão da linguagem é a fala. A voz é definida como a simples emissão sonora produzida pela laringe. A fala é a articulação da voz, produzindo as palavras; é a tradução sonora da linguagem.

Holt $(1969)^{2}$ descreveu sob forma de um esquema a seqüência da linguagem na comunicação humana, ressaltando os processos de decodificação e codificação citados anteriormente (Figura 1). 


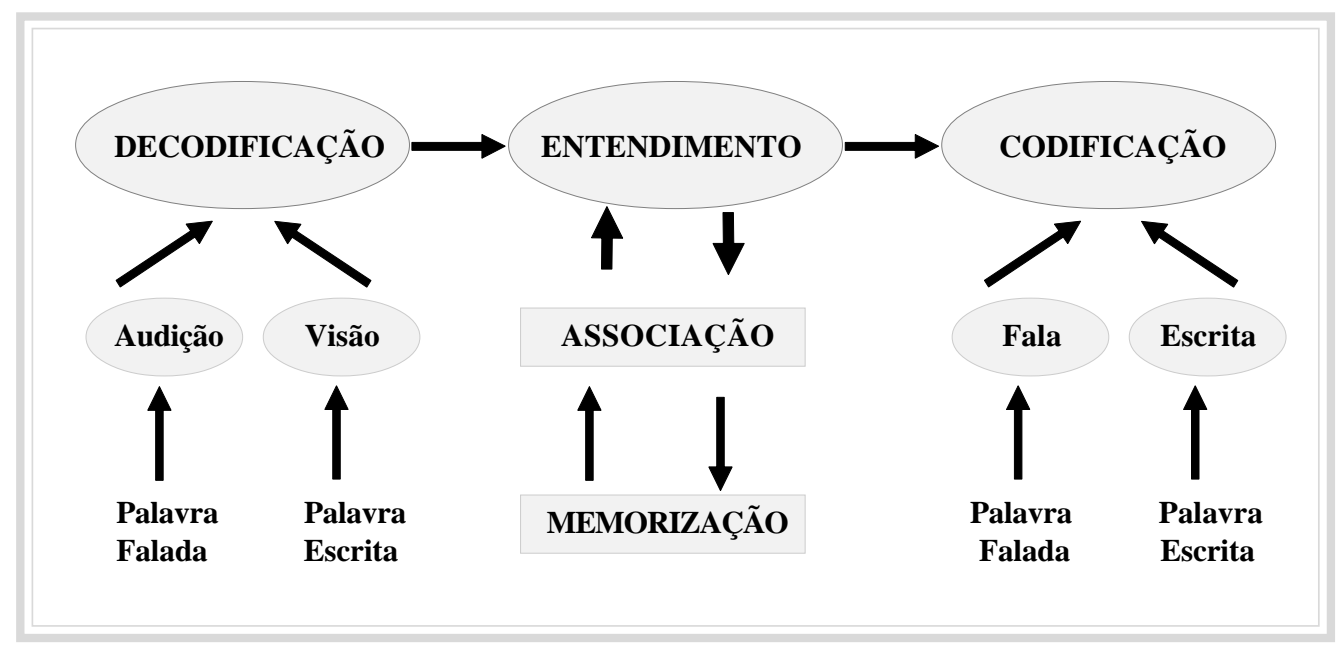

Figura 1: Esquema da seqüência da linguagem².

A comunicação oral é extremamente complexa e utiliza diferentes áreas do córtex cerebral. O hemisfério esquerdo é responsável pelas habilidades de linguagem em $94 \%$ dos adultos destros e $75 \%$ dos adultos sinistros. Neste hemisfério, existem três áreas principais que são especializadas para a linguagem. Na área posterior, ou de Wernicke, ocorre a recepção dos sinais sonoros que codificam a palavra e a comparação com os dados de linguagem armazenados para interpretar o significado; a resposta é formulada e en- viada para as áreas anteriores (área de Broca e córtex motor suplementar), através do fascículo arqueado. Na área de Broca, há a ativação dos programas motores especializados para o controle da formação de palavras. Finalmente, ocorre a transmissão para o córtex motor, a fim de controlar os músculos da fonação e articulação da fala. A especialização hemisférica para a linguagem começa antes do nascimento, mas a função de programação da linguagem dá-se de maneira contínua, através dos anos (Figura 2).

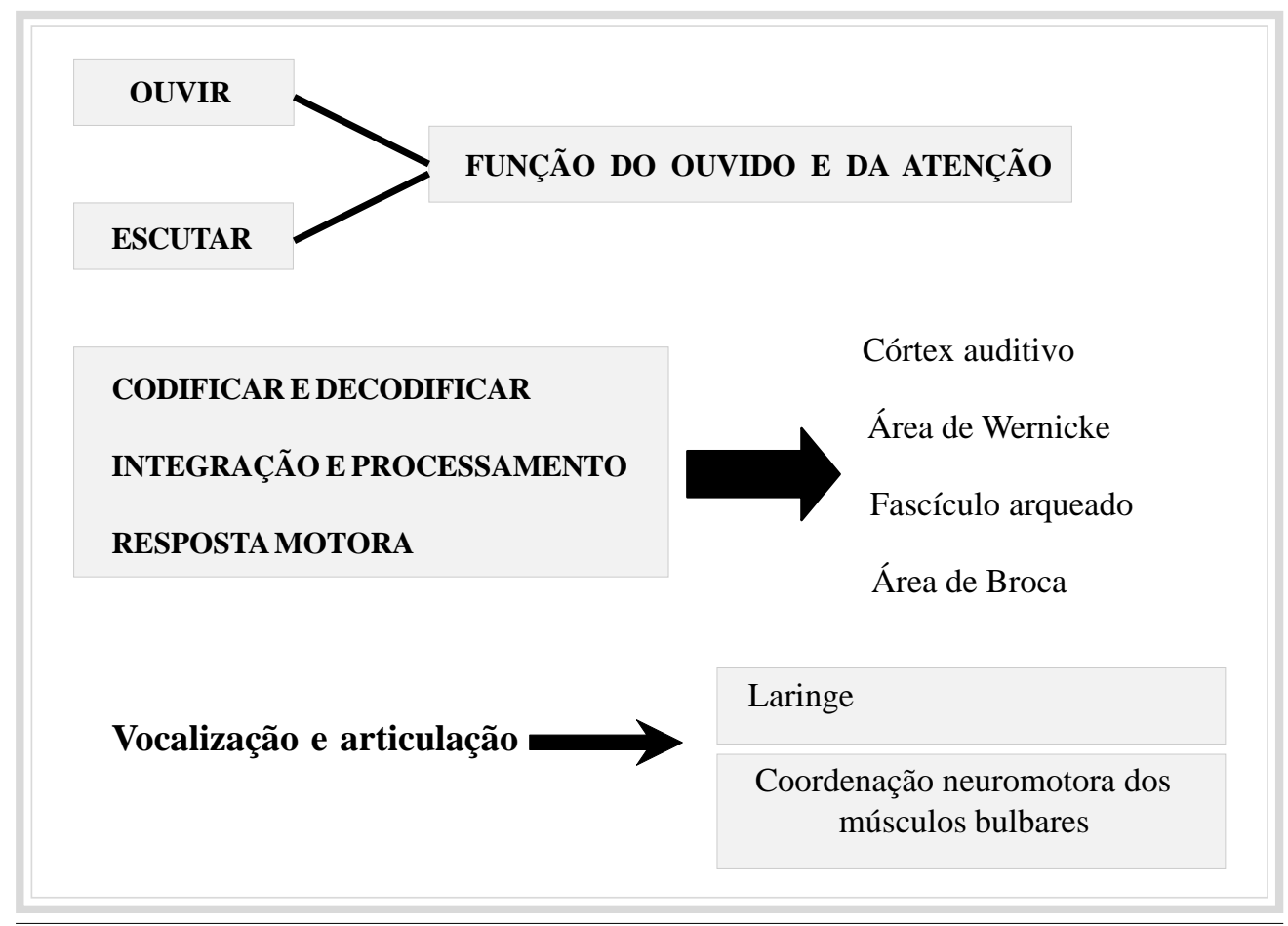

Figura 2: Os passos da comunicação ${ }^{5}$. 
As funções desempenhadas pelo aparelho fonador são:

- respiração: com a fala a inspiração fica mais curta do que a expiração;

- fonação: diz respeito à qualidade e característica da voz produzida pela laringe;

- ressonância: é a modificação seletiva da inflexão da voz, quando a corrente de ar passa através da rinofaringe, orofaringe e da boca;

- articulação: é a habilidade de modificar ou regular a corrente da voz por válvulas como lábios, língua, véu palatino ou faringe, transformando a voz em palavras;

- prosódia: refere-se à qualidade normal da fala, incluindo velocidade de sincronização, intervalo, regulagem, melodia e ênfase. A variação na maneira como a expressão é dada diz respeito à prosódia, ela muda conforme a mudança do tom, intensidade e acentuação que são colocados em lugar específico da expressão.

Para o perfeito funcionamento deste complexo sistema, é preciso que ocorra um sincronismo entre todas as partes do aparelho fonador.

\section{3- ALTERAÇÕES NA COMUNICAÇÃO ORAL}

Quando uma criança é trazida para avaliação com queixa de não falar, em um primeiro contato é muito difícil diagnosticarmos a causa do problema. Devemos partir para uma investigação adequada, e assim poderemos definir se estamos diante de um problema de emissão (fala) ou na formulação, no domínio sobre o código (linguagem). Leung e Kao (1999) ${ }^{3}$ mostraram que a criança é considerada com atraso de fala, quando seu desenvolvimento é abaixo do esperado para uma criança na mesma idade. Sabe-se, porém, que crianças que estão adquirindo a linguagem normalmente podem apresentar variações no seu desenvolvimento, não correspondendo sua performance à de outra criança da mesma idade, e isso pode constituir apenas uma variação do normal.

\section{1- Estágios de expressão da linguagem}

De acordo com Psarommatis e cols. $(2001)^{4}$, a criança começa a se comunicar com o seu meio logo nos primeiros meses de vida. Os estágios de expressão da linguagem e desenvolvimento da fala são:

- de 0 a 8 semanas: choro reflexo e sons vegetativos; - de 2 a 6 meses: monossílabos e sons vogais;
- de 6 a 8 meses: polissílabos e balbucio;

- de 12 a 18 meses: primeiras palavras verdadeiras são usadas, imitação de sons, usa até 20 palavras;

- de 18 a 24 meses: são usadas poucas palavras-frases, vocabulário contendo aproximadamente 200 palavras. As crianças conectam palavras em frases;

- de 24 a 36 meses: formam sentenças combinando 3 a 5 palavras, usam-se plurais;

- de 36 a 48 meses: clara sintaxe, vocabulário de até 1000 palavras, fala completamente inteligível. A criança usa $90 \%$ dos conceitos gramaticais corretamente;

- 5 anos: repertório de 6000 a 10000 palavras;

- 7 anos: reprodução correta de todos os sons da fala.

Embora a aquisição da linguagem e da comunicação desenvolva-se segundo essas etapas constantes, o ritmo de progressão pode divergir de caso a caso, podendo-se esperar uma variação de até seis meses, de acordo com o indivíduo.

\section{2- Diagnóstico dos distúrbios da linguagem}

Aneja (1999) ${ }^{5}$ indica alguns sinais de alerta para desordens da linguagem na criança, tais como: nenhuma palavra emitida até os 18 meses; não colocação de duas palavras juntas aos 2 anos; ausência de desempenho imitativo e simbólico aos 2 anos; não formação de sentenças aos 3 anos; discurso incompreensível aos 3 anos. Oller e cols. (1999) ${ }^{6}$ mostraram que o balbuciar produzido por volta dos 10 meses de idade, quando atrasado, pode prognosticar desordens da fala. Balkwin $(1968)^{7}$ mostra que, por volta dos três anos, a criança já deve se expressar oralmente, entretanto, um leve retardo nesta idade não é incomum, $\mathrm{O}$ atraso na linguagem e/ou fala pode ocorrer três vezes mais em meninos do que em meninas.

Classificar os distúrbios da linguagem que se expressam através das alterações da fala não é tarefa fácil, nem tampouco há consenso na literatura. Diferenciam-se os distúrbios do desenvolvimento da fala e/ou linguagem em orgânicos e não orgânicos. Nos orgânicos, ocorreria uma falha nos órgãos periféricos e/ou no sistema nervoso central. Já os não orgânicos envolveriam questões ambientais e emocionais. O principal problema do falante tardio parece estar no domínio da formulação da linguagem, que tem sido visto como um processo superior ou cognitivo, enquanto a execução é um processo inferior ou motor. 
Podemos ter desarranjos no falar ou no aparelho fonador causados por lesões no sistema nervoso central, no sistema nervoso periférico ou por anormalidades estruturais, levando a um falar anormal, embora a linguagem possa estar normal.

Atrasos da linguagem podem ocorrer devido a vários problemas, tais como: retardo mental, perda auditiva, atraso na maturação, desordem expressiva da linguagem (afasia), bilingüismo, autismo, mutismo eletivo, paralisia cerebral e deprivação psicossocial. Estudos descrevem como sendo a perda auditiva e o retardo mental as duas causas mais freqüentes de dificuldade na aquisição da linguagem e/ou fala.

As habilidades da linguagem e fala dependem da integridade neuromuscular, do sistema sensorial, das influências do meio e das condições emocionais da criança; desta forma, além dos fatores maturativos, torna-se indispensável uma relação adequada e efetiva da criança com o ambiente com o qual ela interage. Na avaliação dos quadros de alteração do desenvolvimento de linguagem e/ou fala, é imprescindível comparar a etapa do desenvolvimento lingüístico com o contexto geral do desenvolvimento sensório-motor e cognitivo da criança, para que se trace uma avaliação global das suas capacidades e aquisições.

Vários autores utilizam o termo Impedimentos Específicos da Linguagem (Specific Language Impairments - SLI) quando se referem a crianças com distúrbios da linguagem cujas dificuldades são específicas dos aspectos linguiísticos, isto é, a alteração da linguagem é primária e não decorrente de uma outra alteração, como deficiência mental, deficiência auditiva, entre outras. Em relação aos quadros de SLI, é importante a diferenciação entre retardo e distúrbio da linguagem. A afirmação de que uma criança tem um retardo da linguagem implica em que comportamentos específicos da linguagem surgem ou se desenvolvem de forma lenta, mas a criança adquire os comportamentos na mesma sequiência observada no desenvolvimento normal, e o grau de retardo é basicamente o mesmo para todos os fatores e aspectos da linguagem. O termo "distúrbio da linguagem" implica em um desvio do padrão usual de aquisição de um ou mais de seus aspectos, em graus variados, interrompendo a sequiência normal do desenvolvimento.

\section{3- Alterações secundárias à perda auditiva}

Como já citado anteriormente, podemos observar que a alteração da linguagem e/ou da fala pode ser secundária, entre outras causas, à perda auditiva.
A audição normal é necessária para que ocorra a aquisição de ambas; o feedback auditivo é de extrema importância no controle da qualidade da fala, sendo, porém, mais importante no seu aprendizado. A identificação da perda auditiva e a intervenção precoce são fundamentais para melhor desenvolvimento da linguagem, pois sabe-se que é alta a incidência de crianças onde a perda auditiva levou a alterações nesse processo.

O desenvolvimento do bebê é altamente dependente da audição, sendo sua adaptação social e intelectual relacionada com o funcionamento normal do sistema auditivo. A deficiência auditiva pré-lingual compromete de forma grave a aquisição da linguagem oral nos primeiros seis a dezoito meses de idade, prejudicando globalmente a comunicação; instalada na fase pós-lingual, em crianças de maior idade, induz a regressão da comunicação oral. A perda do feedback auditivo contribui de maneira marcante para a alteração da elaboração e emissão da fala.

Não apenas os casos de surdez neurossensorial podem levar a alterações no desenvolvimento da linguagem e/ou fala. Vários estudos têm demonstrado que a otite média com efusão, levando a perda auditiva condutiva, pode causar alteração na linguagem. Abraham e cols. (1996) ${ }^{8}$ mostraram que a criança com esse tipo de perda obtém expressivo atraso na linguagem e na aquisição da fala, mostrando menor acurácia e demora no início do uso de consoantes. Pacientes com perda condutiva flutuante têm comprometimento da fala e linguagem, e a habilidade da percepção auditiva deste grupo pode ser menor, mesmo se a função auditiva for normalizada (Schomweiler e cols. 1998) A otite média com efusão durante o desenvolvimento da linguagem pode mudar a sensibilidade auditiva, podendo o indivíduo receber o sinal auditivo parcialmente, ou de forma inconsistente, ou ainda, com perda de informações importantes. Quanto mais cedo a criança for afetada pela otite média secretora, maior é o comprometimento na aquisição das habilidades de linguagem e fala, sabendo-se que é no primeiro ano de vida que se faz a grande discriminação e produção dos sons ${ }^{10}$. Fica, assim, evidente a importância da correção o mais precocemente possível dos casos de alteração de orelha média levando a perda auditiva condutiva em crianças.

\section{4- Influências ambientais}

Além das perdas auditivas, outro aspecto relevante no desenvolvimento da fala e linguagem é a in- 
fluência ambiental ou sociogênica. Mysak (1998) ${ }^{11}$ afirma que desenvolverá a fala mais cedo a criança cujo ambiente propicie rica experiência verbal com adultos e com outras crianças, necessidade real de comunicação oral e os conseqüentes reforços positivos que isso the proporciona. Fatores psicossociais, tais como baixo nível educacional dos pais, distúrbios psiquiátricos dos mesmos, paternidade precoce, famílias incompletas ou com graves problemas de relacionamento são os mais importantes fatores de risco para problemas no desenvolvimento da fala ${ }^{12}$.

Os pais são, geralmente, bons observadores do desenvolvimento da fala das crianças, bem como fundamentais para sua estimulação. Numa simples entrevista, podemos detectar indícios de distúrbios ou atrasos nesse processo, através de informações como:

- há dificuldade para entender as palavras?

- o quanto é possível entender dessas palavras?

- usa gestos?

- a criança escuta? já foi submetida a testes audiológicos?

- houve algum tipo de problema no parto?

- o quanto a criança fala?

- qual o padrão geral de desenvolvimento da criança?

- há história de problemas de fala e/ou linguagem, ou de surdez, na família?

\section{5- Abordagem diagnóstica e terapêutica}

Todas as crianças com história de distúrbios na linguagem e/ou fala devem ser avaliadas, sabendo-se dos vários fatores que circundam essa criança. Comumente, observa-se a somação de causas, tornan- do-se difícil estabelecer uma origem única para o problema. Entretanto, o diagnóstico precoce permite o acompanhamento multidisciplinar e a instituição de medidas terapêuticas eficazes. Desta maneira, médicos e profissionais da área da saúde que lidam com crianças devem estar atentos para este tipo de distúrbio, visando minimizar o impacto do problema no desenvolvimento global desse paciente. Uma vez sendo feito o diagnóstico, o paciente deve ser adequadamente encaminhado, para introdução das condutas terapêuticas o mais rapidamente possível. Neste contexto, os testes audiológicos são fundamentais, e devem ser realizados o mais rapidamente possível, com a finalidade de se detectarem os casos onde a alteração de linguagem e/ou fala foi secundária a perda auditiva. Nestes casos, a reversão do quadro, ou a adaptação de próteses auditivas ou de implante coclear, seguida pela terapia de reabilitação, são de vital importância.

\section{4- CONCLUSÕES}

A queixa de distúrbios da linguagem e/ou fala é muito comum nas crianças, podendo esse distúrbio ser causado por diferentes etiologias, sendo a perda auditiva bastante prevalente em nosso meio.

É necessária uma investigação minuciosa e precoce de todos os fatores que possam estar relacionados à instalação dessa alteração, para um diagnóstico etiológico adequado. Neste contexto, o papel do otorrinolaringologista e do fonoaudiólogo é fundamental, para a detecção de possíveis perdas auditivas e reabilitação precoce.

Vitto MMP, Féres MCLC. Oral communication disturbances in children. Medicina (Ribeirão Preto) 2005; $38(3 / 4): 229-234$

Abstract: Oral communication disturbances in children are very frequent, and can be caused by auditory, neurologic and psychiatric alterations, among others. It's of a great importance to stablish an early diagnosis about the origin of this disturbance, in order to perform the apropriated therapeutic method and to minimize the consequent deficits. The authors present a revision about this theme.

Keywords: Communication Disorders. Language Disorders. Speech Disorders. 


\section{REFERÊNCIAS BIBLIOGRÁFICAS}

1 - Nicolosi L, Harryman E, Kreshck J. Vocabulário das distúrbios da comunicação: fala, linguagem e audição. 3. ed. Porto Alegre: Artes Médicas, 1996.

2 - Holt KS. Language disorder. Proc R Soc Med 1969; 62 (11 Part 1): 1094.

3 - Leung AKC, Kao CP. Evaluation and management of child with speech delay. Am Fam Physician 1999; 59: 3121-8.

4 - Psarommatis IM, Goritsa E, Douniadakis D, Tsakanikos M, Kontrogianni AD, Apostolopoulos N. Hearing loss in speechlanguage delayed children. Int J Pediatr Otorhinolaryngol 2001; 58: 205-10.

5 - Aneja S. Evaluation of a child with communication disorder. Indian Pediatr 1999; 36: 891-900.

6 - Oller DK, Eilers RE, Neal AR, Schwartz HK. Precursors to speech in infancy: the prediction of speech and language disorders. J Commun Disord 1999; 32: 223-45.

7 - Bakwinn HS. Delayed speech - developmental mutism. Pediatr Clin North Am 1968; 15: 627-38.

8 - Abraham SS, Wallace IF, Gravel JS. Early otitis media and phonological development at age 2 years. Laryngoscope 1996; 106: 727-32.

9 - Schönweiler R, Ptok M, Radü HJ. A cross-sectional study of speech and language abilities of children with normal hearing, mild fluctuating conductive hearing loss, or moderate to profound sensorineural hearing loss. Int $\mathrm{J}$ Pediatr Otorhinolaryngol 1998; 44: 251-8.

10 - Roberts JE, Burchinal MR, Davis BP, Collier AM, Henderson FW. Otitis media en early childhood and later language. $J$ Speech Hear Res 1991; 34: 1158-68.

11 - Mysak ED. Patologias dos sistemas da fala. Identificação dos distúrbios da fala, princípios de exame e tratamento. 2. ed. São Paulo: Atheneu, 1998.

12 - Weindricht D, Jennen-Steinmetz C, Laucht M, Esser G, Schmidt $\mathrm{MH}$. At risk for language disorders? Correlates and course of language disorders in preschool children born at risk. Acta Paediatr 1998; 87:1288-94.

\section{BIBLIOGRAFIA RECOMENDADA}

Bloch P. Noções de foniatria. A voz humana. In: Hungria H. Otorrinolaringologia. $7^{\mathrm{a}}$. ed. Rio de Janeiro: Guanabara-Koogan; 1995. p. 186-203.

Brown BB. Language disorders in children. Public Health 1973; 87: $115-8$.

Chapman RS. Processos e distúrbios na aquisição da linguagem. Porto Alegre: Artes Médicas, 1996.

Coplan J. Evaluation of the child with delayed speech or language. Pediatr Ann 1995; 14: 203-8.

Jordal J. Treatment of severe language disorders in children. Folia Phoniatr 1977; 29: 22-60.

Launay C, Borel-Maisomy S. Distúrbios da linguagem, da fala e da voz na infância, 2. ed. São Paulo: Roca, 1989.

Murdoch BE. Desenvolvimento da fala e distúrbios da linguagem. Uma abordagem neuroanatômica e neurofisiológica. Rio de Janeiro: Revinter, 1997.

Rapin I. Children with inadequate language development: management guidelines for otolaryngologists. Int $\mathrm{J}$ Pediatr Otorhinolaryngol 1988; 16: 189-98.

Wing L, Gould J. Severe impairments of social interaction and associated abnormalities in children: epidemiology and classification. J Autism Dev Disord 1979; 9: 11-29. 